\title{
Role of soluble programmed death-1 (sPD-1) and sPD-ligand 1 in patients with cystic echinococcosis
}

\author{
YANHUA LI $^{1}$, YUNFENG XIAO ${ }^{2}$, MINGQUAN SU $^{1}$, RONG ZHANG ${ }^{1}$, \\ JIANBING DING $^{3}$, XIAOKE HAO ${ }^{1}$ and YUEYUN MA ${ }^{1}$
}

\author{
${ }^{1}$ Department of Clinical Laboratory, Xijing Hospital, The Fourth Military Medical University, Xi'an, Shaanxi 710032; \\ ${ }^{2}$ Department of Pharmacy, Tangdu Hospital, The Fourth Military Medical University, Xi'an, Shaanxi 710038; \\ ${ }^{3}$ Xinjiang Laboratory of Hydatid Fundamental Medicine, The First Affiliated Hospital of Xinjiang Medical University, \\ Urumqi, Xinjiang 830000, P.R. China
}

Received February 11, 2015; Accepted October 29, 2015

DOI: $10.3892 /$ etm.2015.2876

\begin{abstract}
The programmed death-1 (PD-1)/PD-ligand 1 (PD-L1) signaling pathway is a negative regulatory mechanism that inhibits $\mathrm{T}$ cell proliferation and cytokine production. Soluble PD-1 (sPD-1) and soluble PD-L1 (sPD-L1), are also involved in regulation of the PD-1/PD-L1 signaling pathway. In the present study, the expression levels of SPD-1 and SPD-L1, as well as those of T helper (Th)1 [including interleukin (IL)-2 and interferon gamma], Th2 (including IL-4, IL-6 and IL-10) and Th17 (including interleukin 17) cell cytokines, were measured in the sera of patients with cystic echinococcosis (CE). Measurements were performed prior to and following after surgery and treatment with cyclic albendazole to investigate the effects of SPD-1 and SPD-L1 in patients with CE. Cytokine expression levels were measured using cytokine bead array and the expression levels of sPD-1 and sPD-L1 were measured using ELISA. In addition, in vitro stimulation was used to detect whether SPD-L1 has a negative regulatory effect on cytokine secretion or homeostasis. The present study observed significantly higher levels of SPD-L1 in patients with CE compared with healthy controls. Significantly elevated levels of Th2 cytokines in the sera of patients with $\mathrm{CE}$ were also observed. The results also suggest that there is an imbalanced expression of Th1 and Th2 cells during CE. In addition, it was demonstrated that SPD-1 and SPD-L1 are regulatory
\end{abstract}

Correspondence to: Professor Yueyun Ma or Professor Xiaoke Hao, Department of Clinical Laboratory, Xijing Hospital, The Fourth Military Medical University, 169 Changle West Road, Xi'an, Shaanxi 710032, P.R. China

E-mail: cmbmayy@fmmu.edu.cn

E-mail: haoxkg@fmmu.edu.cn

Abbreviations: CBA, cytometric bead array; PD-1, programmed death-1; sPD-1, soluble PD-1; PD-L1, PD-ligand 1; sPD-L1, soluble PD-L1; CE, cystic echinococcosis

Key words: soluble programmed death-1, soluble programmed death-ligand 1 , cystic echinococcosis, post-treatment, cytokines factors to the PD-1/PD-L1 signaling pathway, each having opposite effect, suggesting that they regulate the immune response to $\mathrm{CE}$ infection by creating a dynamic balance. In conclusion, SPD-L1 may play an important role in maintaining homeostasis in hosts with CE.

\section{Introduction}

Cystic echinococcosis (CE), a chronic helminthic disease that is caused by infection with metacestodes of the tapeworm Echinococcus granulosus, is one of the most widespread zoonotic diseases in humans in developing and developed countries (1). CE is prevalent in temperate zone countries, including South America, the entire Mediterranean region, Russia, central Asia, China and parts of Africa (2-4). In China, primary echinococcosis has been recorded in 25 different regions and is mainly endemic in pasturing areas (such as Xinjiang, Qinghai, Gansu and Ningxia) and semi-pasturing areas (5). Typically, CE patients survive for a number of years without requiring treatment. The common treatment strategy in resource-poor areas includes surgery followed by treatment with cyclic albendazole and regular follow-up (6). Albendazole is a broad-spectrum anthelmintic that has been used in the treatment of hydatid disease, in combination with surgery $(6,7)$. The management of $\mathrm{CE}$ infection requires various components or systemic factors in order to maintain homeostasis in patients hosting Echinococcus granulosus. Generally, levels of serum cytokines are used as an indication of homeostasis status $(8,9)$.

Costimulatory molecules serve a crucial function in the long-term interaction between the parasite and the host in CE. For example, the negative co-stimulatory molecule programmed death-1 (PD-1) and its ligands, PD-ligand-1 (PD-L1; also known as B7-H1) and PD-ligand 2 (PD-L2; also known as B7-DC), negatively regulate anti-infection pathways $(10,11)$. These can inhibit or downregulate $\mathrm{T}$ cell activation, cytokine production and pathogen elimination, and can also enhance host resistance to pathogens, thereby promoting the suppression of the immune system (12-14). The PD-1 molecule is an inhibitory receptor that is induced and activated by CD 28 and CTLA- 4 cell surface receptors, which are predominantly expressed in activated T cells (15). CD28 
is required as an effector for regulatory $\mathrm{T}$ cell differentiation. Effective regulatory T cells express CTLA-4 and PD-1, which function as inhibitory receptors. PD-L1 and PD-L2 are primarily expressed on dendritic cells. Activation of PD-1 receptors may negatively regulate $\mathrm{T}$ cell receptor signaling and reduce the proliferation and production of cytokines; however, it may stimulate the production of the interleukin-10 (IL-10) cytokine.

PD-1 and PD-L1 also exist in soluble forms, namely soluble PD-1 (sPD-1) and soluble PD-L1 (sPD-L1) (16). sPD-1 is encoded by the PD-1 splice variant, PD-1 $1 \Delta$ ex 3 , and SPD-Ll can be detected in physiological and pathological conditions. SPD-Ll is produced when matrix metalloproteinase enzymes cleave the extracellular fraction of membrane type PD-L1 cells (mPD- $\mathrm{Ll}^{+}$) (17). sPD-1 and sPD-Ll are known to have opposite regulatory effects. sPD-1 inhibits the PD-1/PD-L signaling pathway by interacting with PD-L1, and therefore promotes the activation of T cells. However, SPD-L1 binds to the PD-1 receptor on the surface of the T cell, which produces an inhibitory signal, thereby inhibiting $\mathrm{T}$ cell activation and proliferation. Although SPD-1 and SPD-Ll have been recognized as naturally existing regulators of PD-1/PD-L1 membrane signaling pathways in various disease systems, the effects of sPD-1 and SPD-L1 regulation in CE has not been reported. In addition, the association between albendazole and PD-1/PD-L1 remains unclear The present study investigated the role of SPD-1 and SPD-L1 in CE by measuring the levels of sPD-1, sPD-L1 and cytokines in the sera of patients with $\mathrm{CE}$ using cytometric bead array (CBA) and enzyme-linked immunosorbent assay (ELISA).

\section{Materials and methods}

Instruments and reagents. The xMark microplate absorbance spectrophotometer was purchased from Bio-Rad Laboratories, Inc. (Philadelphia, PA, USA) and a HydroFlex microplate washer was purchased from Tecan US, Inc. (Morrisville, NC, USA). human SPD-1 and sPD-L1 ELISA kits were purchased from Wuhan Sanying Biotechnology (Wuhan, China) and the BD CBA Human Th1/Th2/Th17 Cytokine kit was purchased from BD Biosciences (cat. no. 560484; San Jose, CA, USA.). sPD-L1 was provided by the Institute of Tissue Transplantation and Immunology, College of Life Science and Technology, Jinan University (Guangzhou, China). RPMI-1640 (Thermo Fisher Scientific, Inc., Waltham, MA, USA) was used according to the manufacturer's instructions, after preparing the filtration, subpackaging and the cryopreservation standby. Fetal bovine serum (FBS) was purchased from Thermo Fisher Scientific, Inc., and was frozen and inactivated at $56^{\circ} \mathrm{C}$. Phytohemagglutinin (PHA; Sigma-Aldrich China Inc., Shanghai, China) was used according to the manufacturer's instructions. Penicillin $(50,000 \mathrm{U} / \mathrm{ml})$, streptomycin $(50,000 \mu \mathrm{g} / \mathrm{ml})$ and sterile physiological saline were purchased from Beyotime Institute of Biotechnology (Shanghai, China). The final concentration of the RPMI-1640 culture medium, containing sPD-L1, antibiotics and PHA, was $100 \mathrm{U} / \mathrm{ml}$.

Patients and healthy controls. Healthy subjects and patients with CE were enrolled into this study after obtaining signed informed consent, and the study followed the protocols approved by the Institutional Review Board of the Xinjiang Medical University (Xinjiang, China; approval no. 20120220-126). A total of 51 patients with CE, including 29 male and 22 female patients from The First Affiliated Hospital of Xinjiang Medical University (Xinjiang, China), were enrolled in the study between December 2009 and December 2012. Patient ages ranged between 21 and 58 years, with a median age of 37 years. The majority of patients with $\mathrm{CE}$ were from agricultural and pastoral areas. In addition, 20 healthy volunteers of matched age and gender were recruited from The First Affiliated Hospital of Xinjiang Medical University as healthy control subjects. All the patients with preoperative $\mathrm{CE}$ were diagnosed according to self-reported symptoms (including nausea, abdominal distension/pain and weight loss), physical examination and type B ultrasonic imaging. The presence of liver CE in patients was confirmed by surgery, preoperative examination (including computerized tomography and magnetic resonance imaging) and pathological diagnosis. Patients underwent appropriate surgery, such as internal or external capsule cavity drainage, followed by treatment with $10-15 \mathrm{mg} / \mathrm{kg}$ per day albendazole (Shaanxi Hanwang Chinese Medicine Co., Ltd., Shaanxi, China) for six months, as described in a previous study by Li et al (6).

Sample preparation. Blood samples $(3 \mathrm{ml})$ were collected from patients and healthy subjects during outpatient examination, and incubated in a water bath at $37^{\circ} \mathrm{C}$ for $30 \mathrm{~min}$. Blood samples were then centrifuged at 4,000 $\mathrm{x}$ g for $5 \mathrm{~min}$, and the supernatants were collected into $1 \mathrm{ml}$ Eppendorf tubes as serum samples and stored at $-80^{\circ} \mathrm{C}$ in a refrigerator until use.

Measurement of SPD-1 and SPD-L1 in sera. Levels of SPD-1 and SPD-L1 in the sera of CE patients and healthy volunteers were detected using an ELISA kit, following the manufacturer's instructions. Briefly, blood samples were incubated at $37^{\circ} \mathrm{C}$ for $30 \mathrm{~min}$, then centrifuged for $5 \mathrm{~min}$ at $4,000 \mathrm{x}$ g (room temperature) and the sera were collected. Next, horseradish peroxidase (HRP)-conjugated SPD-1 antibody was added to each serum sample to form an antigen-antibody complex. Substrate (3,3',5,5'-tetramethylbenzidine) appeared blue under the catalysis of HRP, and changed to yellow in the presence of acid. The color depth and SPD-1 in the sample were positively correlated. Absorbance (optical density) was measured at $450 \mathrm{~nm}$, and a standard curve was produced to calculate the concentration of SPD-1.

Measurement of cytokines in sera. A CBA Human Th1/Th2/Th17 Cytokine kit was used to measure the T helper (Th) 1 [including interleukin (IL)- 2 and interferon- $\gamma$ (IFN- $\gamma$ )], Th2 [including IL-4, IL-6, and IL-10] and Th17 cytokines [including IL-17] expression levels in the sera of healthy controls and patients (pretreatment and post-treatment). The kit performance was optimized for the analysis of physiologically relevant $(\mathrm{pg} / \mathrm{ml})$ specific cytokine proteins in tissue culture supernatants and serum samples. All samples were incubated for $30 \mathrm{~min}$ at room temperature. During the assay procedure, the cytokine capture beads were mixed with recombinant Human Soluble Protein Flex Set Standard and 
Table I. Relative levels of IL-2, IFN- $\gamma$, IL-4, IL-10, IL-6 and IL-17 in serum samples.

\begin{tabular}{|c|c|c|c|c|c|c|c|}
\hline \multirow[b]{2}{*}{ Group } & \multirow[b]{2}{*}{$\mathrm{n}$} & \multicolumn{3}{|c|}{ Th1 } & \multicolumn{2}{|c|}{ Th2 } & \multirow{2}{*}{$\frac{\mathrm{Th} 17}{\mathrm{IL}-17(\mathrm{pg} / \mathrm{ml})}$} \\
\hline & & $\mathrm{IL}-2(\mathrm{pg} / \mathrm{ml})$ & $\mathrm{IFN}-\gamma(\mathrm{pg} / \mathrm{ml})$ & $\mathrm{IL}-4(\mathrm{pg} / \mathrm{ml})$ & $\mathrm{IL}-10(\mathrm{pg} / \mathrm{ml})$ & IL-6 (pg/ml) & \\
\hline $\mathrm{HC}$ & 20 & $2.85 \pm 0.41$ & $2.78 \pm 0.48$ & $1.80 \pm 0.15$ & $10.12 \pm 2.07$ & $4.36 \pm 1.70$ & $27.33 \pm 1.70$ \\
\hline $\mathrm{CE}$ & 51 & $2.60 \pm 0.30$ & $2.94 \pm 0.31$ & $2.06 \pm 0.38^{a}$ & $22.53 \pm 6.63^{b}$ & $8.95 \pm 7.79^{\mathrm{a}}$ & $28.55 \pm 2.45$ \\
\hline AT & 45 & $2.96 \pm 0.53$ & $2.81 \pm 0.73$ & $1.91 \pm 0.24$ & $11.32 \pm 3.15$ & $5.44 \pm 2.47$ & $27.43 \pm 3.27$ \\
\hline
\end{tabular}

${ }^{\mathrm{a}} \mathrm{P}<0.05$ and ${ }^{\mathrm{b}} \mathrm{P}<0.01$ vs. control. Only 45 of the initial 51 patients are included in the AT group as 6 patients were lost to follow-up. Th, helper T cell; IL, interleukin; IFN- $\gamma$, interferon- $\gamma$; HC, healthy control; CE, cystic echinococcosis; AT, after-treatment CE patients.
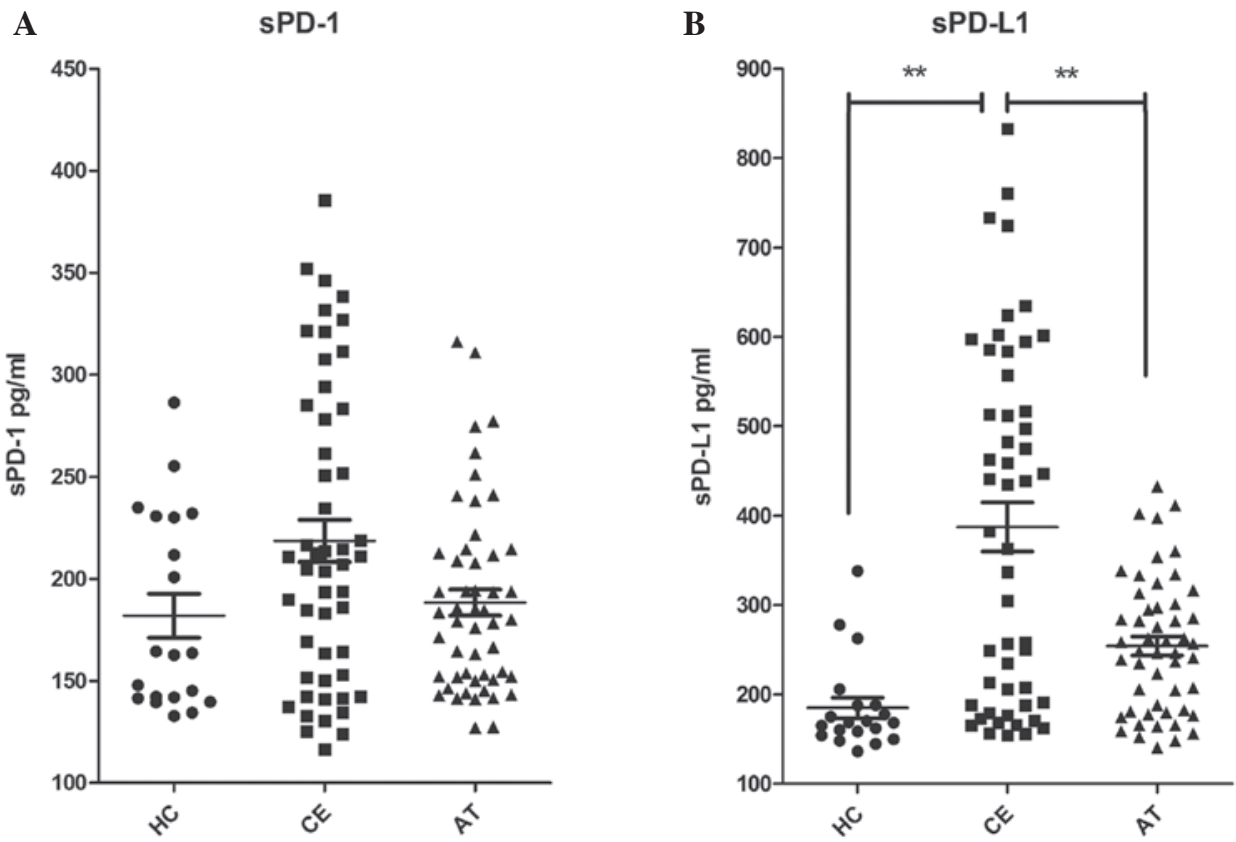

Figure 1. Levels of SPD-1 and SPD-L1 increased in patients with CE and reduced after surgery and 6-month treatment with cyclic albendazole. Concentrations of (A) SPD-1 and (B) SPD-L1 in serum samples from HC (filled circles), CE (filled squares) and AT (filled triangles) were measured by ELISA. Each spot represents one study subject and lines indicate the mean \pm standard deviation. ${ }^{* *} \mathrm{P}<0.01$, comparison shown by brackets. sPD-1, soluble programmed death-1; sPD-L1, sPD-ligand 1; HC, healthy control; CE, cystic echinococcosis; AT, after-treatment CE patients.

samples, then incubated for $3 \mathrm{~h}$ with the rabbit phycoerythrin (PE)-conjugated detection antibodies (included with the Th1/Th2/Th17 cytokine kit; cat. no. 560484) to form sandwich complexes, then centrifuged for $5 \mathrm{~min}$ at $600 \mathrm{x} \mathrm{g}$. The supernatant was then discarded and resuspended with $300 \mu \mathrm{l}$ phosphate-buffered saline. The intensity of the PE fluorescence of each sandwich complex revealed the concentration of the cytokine present, as previous described (18). After measuring the samples using a flow cytometer (FACSCalibur; BD Biosciences, Franklin Lakes, NJ, USA), FCAP Array software (BD Biosciences) was used to generate results in graphical and tabular format in accordance with the operating instructions. A standard curve was used to calculate the cytokine levels.

Measurement of $T$ cell proliferation and cytokine production stimulated by $S P D-L 1$ in vitro. Peripheral blood mononuclear cells from 11 patients, selected at random from the 51 patients with CE prior to treatment, were cultured in RPMI-1640 medium supplemented with antibiotics and 10\% FBS (total volume, $200 \mu 1 ; 2 \times 10^{6} / \mathrm{ml}$ in a 96 -well plate). Next, $\sim 95 \%$ of the cells were stimulated with PHA $(1 \mu \mathrm{g} / \mathrm{ml})$, while $274 \%$ of the cells were stimulated with PHA in addition to $0.4 \mathrm{ng} / \mathrm{ml} \mathrm{sPD}-\mathrm{L} 1$. At $24 \mathrm{~h}$ post-incubation, the culture supernatants were collected by centrifugation at $1,000 \mathrm{x} \mathrm{g}$ for $10 \mathrm{~min}$ at room temperature, and stored at $-80^{\circ} \mathrm{C}$ before the levels of cytokines were measured. To evaluate $\mathrm{T}$ cell proliferation, $10 \mu 1$ MTT $(5 \mathrm{mg} / \mathrm{ml})$ was added to each well $6 \mathrm{~h}$ before the end of the culturing period. The proliferation of $\mathrm{T}$ cells was measured based on the production of insoluble MTT metabolites by live cells.

Statistical analysis. Statistical analyses were performed and graphs were constructed using SAS version 3.0 (SAS Institute Inc., Cary, NC, USA), SPSS software version 17.0 (SPSS, Inc., Chicago, IL, USA) and Prism version 5 software (GraphPad Software, Inc., La Jolla, CA, USA). Experimental data were analyzed using independent samples t-test, Pearson correlation analysis and multiple comparisons. $\mathrm{P}<0.05$ was considered to indicate a statistically significant difference. 
Table II. Relative levels of IL-2, IFN- $\gamma$, IL-4, IL-10, IL-6 and IL-17 after stimulation with sPD-L1 in vitro.

\begin{tabular}{|c|c|c|c|c|c|c|c|c|}
\hline \multirow[b]{2}{*}{ Group } & \multirow[b]{2}{*}{$\mathrm{r}$} & \multirow[b]{2}{*}{$\mathrm{n}$} & \multicolumn{3}{|c|}{ Th1 } & \multicolumn{2}{|c|}{ Th2 } & \multirow{2}{*}{$\frac{\text { Th17) }}{\mathrm{IL}-17(\mathrm{ng} / \mathrm{ml})}$} \\
\hline & & & $\mathrm{IL}-2$ (ng/ml) & $\mathrm{IFN}-\gamma(\mathrm{ng} / \mathrm{ml})$ & IL-4 (ng/ml) & IL-10 (ng/ml) & IL-6 (ng/ml) & \\
\hline G & 95 & 11 & $2.87 \pm 0.26$ & $2.79 \pm 0.28$ & $1.92 \pm 0.25$ & $19.45 \pm 5.27$ & $8.28 \pm 4.35$ & $27.93 \pm 2.57$ \\
\hline sPG & 74 & 11 & $2.35 \pm 0.42$ & $2.68 \pm 0.59$ & $2.13 \pm 0.32^{\mathrm{a}}$ & $24.46 \pm 3.33^{\mathrm{b}}$ & $9.68 \pm 3.17^{\mathrm{b}}$ & $28.29 \pm 2.71$ \\
\hline
\end{tabular}

${ }^{\mathrm{a}} \mathrm{P}<0.01$ and ${ }^{\mathrm{b}} \mathrm{P}<0.05$ vs. CG. IL, interleukin; IFN- $\gamma$, interferon $\gamma$; sPD-L1, soluble programmed death-ligand 1 ; Th, helper T cell; r, relative growth rate $(\%)$ relative to all cells in medium; CG, without stimulation by sPD-L1; sPG, with stimulation by sPD-L1.

\section{Results}

Elevated levels of $S P D-1$ and $S P D-L 1$ in patients with $C E$. To explore the roles of SPD-1 and SPD-L1 in the pathogenesis of $\mathrm{CE}$, the concentrations of SPD-1 and SPD-L1 were measured in the patient sera and compared with those measured in the sera of the healthy subjects. The expression of sPD-1 was not found to be significantly higher in the sera of patients with $\mathrm{CE}$ prior to treatment $(218.6 \pm 73.4 \mathrm{pg} / \mathrm{ml})$ compared with that in healthy subjects $(181.8 \pm 48.2 \mathrm{pg} / \mathrm{ml} ; \mathrm{P}=0.053$; Fig. 1A). In addition, no significant changes were observed in patients with $\mathrm{CE}$ after treatment with albendazole for 6 months $(209.9 \pm 67.6 \mathrm{pg} / \mathrm{ml}$; Fig. 1A). The expression of sPD-L1 in the sera of patients with CE prior to treatment $(387.5 \pm 196.5 \mathrm{pg} / \mathrm{ml})$ was significantly increased compared with that in healthy controls $(185.0 \pm 51.0 \mathrm{pg} / \mathrm{ml} ; \mathrm{P}<0.001$; Fig. 1B), while the expression decreased sharply after treatment $(254.3 \pm 75.0 \mathrm{pg} / \mathrm{ml} ; \mathrm{P}<0.01$; Fig. 1B). These results demonstrate that SPD-L1 levels are significantly higher in patients with CE compared with those in healthy subjects.

Cytokines in the sera of patients with CE. To examine whether changes in the serum levels of SPD-1 and SPD-L1 in patients with $\mathrm{CE}$ correlated with changes in the levels of cytokines, the concentrations of IL-2, IFN- $\gamma$, IL-4, IL-10, IL-6 and IL-17 in the sera were measured using a CBA. The results, as summarized in Table I, demonstrated that the levels of IL-2 and IFN- $\gamma$ in the sera of patients with CE showed no significant difference compared with their levels in the healthy controls. However, the cytokines IL-4 ( $\mathrm{P}=0.023)$, IL-6 ( $\mathrm{P}=0.014)$ and IL-10 $(\mathrm{P}<0.01)$ in the patients sera were significantly increased compared with those subjects in healthy control. IL-17 levels in the sera of patients with CE prior to treatment were found to be increased, but not significantly, compared with the healthy control subjects. Subsequent to surgery and 6-month treatment with cyclic albendazole, the levels of IL-17 decreased, returning to the control levels. This may be due to Th2 cells proliferating and differentiating into Th17 cells, in addition to the high levels of IL-10 secreted by Th2 cells. IL-10 is considered to be an effective anti-inflammatory factor, and is able to inhibit immune response mediated by Th1 and Th17. Furthermore, the functions of sPD-L1 in regulating Th1, Th2 and Th17 during echinococcosis have been suggested.

Effect of $S P D-L 1$ on lymphocyte cytokine production and proliferation. To examine the physiological significance of SPD-L1 in disease development, the effect of sPD-L1 on T cell cytokine production and proliferation was observed in vitro. The results, as summarized in Table II, demonstrated that the addition of SPD-L1 significantly increased the production of T cell cytokines, IL-4, IL-6 and IL-10, and significantly inhibited the production of IL-2, compared with cells without sPD-L1 stimulation $(\mathrm{P}<0.05)$. No significant effects were measured on IFN- $\gamma$ or IL-17. These results are consistent with those of previous studies in other diseases (19), suggesting that SPD-L1 inhibits Th1 immune responses and supports the Th2 immune response, resulting in a stronger Th2 immune response.

\section{Discussion}

Hydatid disease (hydatidosis), also known as echinococcosis, is a zoonotic disease caused by the parasite Echinococcus granulosus, which affects humans and other animals globally, and can cause severe damage to the host $(20,21)$. A long-term balanced interaction between the host and the parasite must be established through the complex regulation of host immune responses to reduce damage. Negative co-stimulatory molecules, such as PD-1, play a critical role in this process, for example by helping antigens evade a host immune attack (22). In turn, sPD-1 and sPD-L1 perform regulatory roles in physiological and pathological conditions by affecting the PD-1/PD-L1 signaling pathway (23).

SPD-l inhibits the PD-1/PD-L signaling pathway primarily by interacting with the cell surface molecule, PD-L. sPD-L1 is produced when matrix metalloproteinases cleave the membrane PD-L1 molecule on PD-L1 expressing cells $\left(\mathrm{mPD}-\mathrm{Ll}^{+}\right)$. sPD-L1 binds to membrane-bound PD-1, creating a negative signal, which inhibits the activation and proliferation of $\mathrm{T}$ cells. The results in the present study showed that, compared with the healthy controls, the concentration of SPD-1 in the serum of patients with CE was slightly elevated compared with the healthy control subjects and CE patients that received treatment. This increase in SPD-1 expression inhibits the PD-1/PD-L1 signaling pathway in $\mathrm{T}$ cells through negative feedback, which then reduces the inhibition of $\mathrm{T}$ cell activation and increases the activity of the immune system in managing CE. This supports the findings of Wang et al, which suggest that $\mathrm{SPD}-1$ blocks the membrane PD-1 binding site on activated T-cells, thereby attenuating the PD-1 signaling pathway and increasing the immune response (24).

However, in the present study, the concentration of sPD-1 in the serum of patients with $\mathrm{CE}$ was found to be lower than the concentration of SPD-L1, which increased significantly 
compared with the healthy control subjects and CE patients after treatment. This may be due to the fact that, without any treatment for $\mathrm{CE}$, the immune response was compromised, although still functioning to combat the chronic infectious disease. This supports the results showing a small increase in SPD-1 expression levels, but a significant increase in the immune-suppressing SPD-L1 expression levels.

In the present study, sPD-1 and sPD-L1 expression levels decreased after CE patients underwent surgery and treatment with cyclic albendazole. This may be due to the clearance of parasites and reduced pathogenic stimulation as a result of reducing/eliminating the infection. sPD-1 and sPD-L1 have opposite effects on the PD-1/PD-L signaling pathway, and create a dynamic balance in the immune system. Any imbalances in this regulatory mechanism may dysregulate the immune responses. The expression of sPD-1 is known to inhibit PD-L1 in various cell types in mice $(23,25)$, including molecules expressing PD-L1 as a result of a CE infection and PD-L1 molecules associated with $\mathrm{T}$ cell apoptosis, thereby increasing the secretion of cytokines.

As sPD-L1 spreads throughout the body via the blood and lymphatic circulation, it exerts a widespread inhibitory effect by interacting with cell surface receptors, resulting in SPD-L1 reaching far beyond membrane-bound PD-1. This is consistent with the results of the in vitro stimulation tests of the current study, which demonstrated that $\mathrm{T}$ cells from $\mathrm{CE}$ patients that were stimulated by SPD-L1 secreted more IL-10 and less IL-2 compared with CE patients that were not stimulated by SPD-L1. Thus, the parasite antigen-specific $\mathrm{T}$ cells cannot be detected in time to sufficiently remove the antigens, resulting in immune escape of $\mathrm{CE}$ in the body. Therefore, the level of sPD-L1 expression may serve a useful function in predicting the efficacy of treatment and the prognosis of CE.

The CBA results in the present study suggest that there may be an imbalance between the activation of Th1 and Th2 during the development of $\mathrm{CE}$. The present study confirmed that patients with $\mathrm{CE}$ demonstrated predominantly increased expression of Th2 cytokines, such as IL-4, IL-10 and IL-6, compared with Th1 cytokines, during chronic CE infection, increasing immunity against the partially inhibited cytotoxicity reaction. Riganò et al (26) demonstrated that, with a gradually increasing parasite load, the immune response activates the humoral immunity of Th2 cells and promotes the secretion of IL-4 and IL-10 in CE patients. This has also been observed in secondary echinococcosis in mice at an advanced stage of Echinococcus infection (27-29).

The present study demonstrated that, compared with healthy subjects, IL-10 and IL-6 expression increased significantly in patients with CE and IL-2 expression decreased, whereas there was no significant change in the expression of IFN- $\gamma$. During a CE infection, an imbalance between Th1 and Th2 cells occurs, with Th2 cells dominating; however, this does not totally eliminate the function of Th1 immune response. The present results also demonstrated that IL-17 secretion did not change significantly in patients with $\mathrm{CE}$, which may be due to Th2 cells differentiating and proliferating to Th17 cells (30). Furthermore, the expression of IL-10, secreted by Th2 cells, greatly increased in patients with CE. IL-10 is considered to be an effective anti-inflammatory factor and can inhibit immune responses mediated by Th1 and Th17 (31). This may explain why no significant changes were observed in Th17 expression in patients with CE. Lechner et al (32) described how proper immune regulation, in response to an $\mathrm{CE}$ infection, depends on IL-17 regulation in pro-inflammatory immune responses. This can promote parasite tissue infiltration and long-term growth in vivo, and can result in immune escape in a host of echinococcosis.

In the present study, after treatment with cyclic albendazole for 6 months, the serum cytokines in patients treated for $\mathrm{CE}$ helped maintain homeostasis by interacting with sPD-1 and sPD-L1. The current study examined the expression levels of sPD-1, sPD-L1, Th1 (IL-2 and IFN- $\gamma$ ), Th2 (IL-4, IL-6 and IL-10) and Th17 (IL-17) prior to and subsequent to surgery followed by cyclic albendazole treatment in patients with $\mathrm{CE}$. Furthermore, in vitro stimulation with sPD-L1 confirmed that sPD-L1 contributes towards the regulation of Th1, Th2 and Th17 in CE. To the best of our knowledge, the current study is the first to demonstrate that an elevated concentration of serum SPD-L1 may have negative regulatory effects in patients with CE. sPD-1 and sPD-L1, as a pair of opposing regulatory factors to the PD-1/PD-L signaling pathway, may participate in immune regulation by creating a dynamic balance. An unregulated interaction of these factors may cause an imbalance in immune responses to an $\mathrm{CE}$ infection. Therefore, by controlling the expression of sPD-L1, it may be possible to block the inhibitory effect of the PD-1/PD-L1 signaling pathway and improve the function of effector $\mathrm{T}$ cells. Controlling the expression of sPD-L1 may therefore be a potentially attractive treatment strategy for managing echinococcosis.

\section{Acknowledgements}

The present study was supported by grants from the National Natural Science Foundation of China (nos. 81160200 and 81371857).

\section{References}

1. Siracusano A, Delunardo F, Teggi A and Ortona E: Host-parasite relationship in cystic echinococcosis: An evolving story. Clin Dev Immunol 2012: 639362, 2012.

2. Yang YR, Sun T, Li Z, Zhang J, Teng J, Liu X, Liu R, Zhao R, Jones MK, Wang Y, et al: Community surveys and risk factor analysis of human alveolar and cystic echinococcosis in Ningxia Hui Autonomous Region, China. Bull World Health Organ 84: 714-721, 2006.

3. Moro PL and Schantz PM: Echinococcosis: Historical landmarks and progress in research and control. Ann Trop Med Parasitol 100: 703-714, 2006.

4. Dakkak A: Echinococcosis/hydatidosis: A severe threat in Mediterranean countries. Vet Parasitol 174: 2-11, 2010.

5. Nunnari G, Pinzone MR, Gruttadauria S, Celesia BM, Madeddu G, Malaguarnera G, Pavone P, Cappellani A and Cacopardo B: Hepatic echinococcosis: Clinical and therapeutic aspects. World J Gastroenterol 18: 1448-1458, 2012.

6. Li T, Ito A, Pengcuo R, Sako Y, Chen X, Qiu D, Xiao N and Craig PS: Post-treatment follow-up study of abdominal cystic echinococcosis in tibetan communities of northwest Sichuan Province, China. PLoS Negl Trop Dis 5: e1364, 2011.

7. Armiñanzas C, Gutiérrez-Cuadra M and Fariñas MC: Hydatidosis: Epidemiological, clinical, diagnostic and therapeutic aspects. Rev Esp Quimioter 28: 116-124, 2015 (In Spanish).

8. Costamagna D, Costelli P, Sampaolesi M and Penna F: Role of inflammation in muscle homeostasis and myogenesis. Mediators Inflamm 2015: 805172, 2015.

9. Cheng LS, Liu Y and Jiang W: Restoring homeostasis of CD4(+) $\mathrm{T}$ cells in hepatitis-B-virus-related liver fibrosis. World J Gastroenterol 38: 10721-10731, 2015. 
10. Hafalla JC, Claser C, Couper KN, Grau GE, Renia L, de Souza JB and Riley EM: The CTLA-4 and PD-1/PD-L1 inhibitory pathways independently regulate host resistance to Plasmodium-induced acute immune pathology. PLoS Pathog 8: e1002504, 2012.

11. Hofmeyer KA, Jeon H and Zang X: The PD-1/PD-L1 (B7-H1) pathway in chronic infection-induced cytotoxic $\mathrm{T}$ lymphocyte exhaustion. J Biomed Biotechnol 2011: 451694, 2011.

12. Ghiotto M, Gauthier L, Serriari N, Pastor S, Truneh A, Nunès JA and Olive D: PD-L1 and PD-L2 differ in their molecular mechanisms of interaction with PD-1. Int Immunol 22: 651-660, 2010

13. McGrath MM and Najafian N: The role of coinhibitory signaling pathways in transplantation and tolerance. Front Immunol 3: 47, 2012.

14. Li Y, Zhu Y, Zhu M and Ding J: The expression and balance of ICOS and PD-1 on the surface of Tregs during infectious diseases. Xi Bao Yu Fen Zi Mian Yi Xue Za Zhi 28: 1229-1231, 2012 (In Chinese).

15. Zhang R, Borges CM, Fan MY, Harris JE and Turka LA: Requirement for CD28 in effector regulatory T Cell Differentiation, CCR6 induction, and skin homing. J Immunol 195 4154-4161, 2015.

16. Hu Z, Yongjing C, Wang Q, Shi B, Bai L and Zhang X: Expression of human PD-1 $\triangle$ ex 3 and primary study of its biological activity. Xi Bao Yu Fen Zi Mian Yi Xue Za Zhi 26: 207-210, 2010 (In Chinese)

17. Chen Y and Zhang X: The role of soluble PD-1 and PD-L1 in the regulation of PD-1/PD-L1 inhibitory pathway and their biological significance. PhD dissertation, Suzhou University. China Doctoral Dissertations Database, 2010 (In Chinese)

18. Zhang H, Hu K, Lv G, Fu Y, Ayana DA, Zhao P and Jiang Y: The imbalance between Tregs, Th17 cells and inflammatory cytokines among renal transplant recipients. BMC Immunol 16: $56,2015$.

19. Singh A, Dey AB, Mohan A, Sharma PK and Mitra DK: Foxp3+ regulatory $\mathrm{T}$ cells among tuberculosis patients: Impact on prognosis and restoration of antigen specific IFN- $\gamma$ producing T cells. PLoS One 7: e44728, 2012.

20. Eckert J and Deplazes P: Biological, epidemiological, and clinical aspects of echinococcosis, a zoonosis of increasing concern. Clin Microbiol Rev 17: 107-135, 2004.

21. Rahimi HR, Sarkari B, Mohammadzadeh T and Sadjjadi SM Immune responses to antigens of in vitro reared Echinococcus granulosus adult worms in Balb/c mice. Iran J Immunol 8 : 236-243, 2011.
22. Horne-Debets JM, Faleiro R, Karunarathne DS, Liu XQ, Lineburg KE, Poh CM, Grotenbreg GM, Hill GR, MacDonald KP, Good MF, et al: PD-1 dependent exhaustion of CD8+ T cells drives chronic malaria. Cell Rep 5: 1204-1213, 2013.

23. Hatachi S, Iwai Y, Kawano S, Morinobu S, Kobayashi M, Koshiba M, Saura R, Kurosaka M, Honjo T and Kumagai S: $\mathrm{CD} 4^{+} \mathrm{PD}-1^{+} \mathrm{T}$ cells accumulate as unique anergic cells in rheumatoid arthritis synovial fluid. J Rheumatol 30: 1410-1419, 2003.

24. Wang D, Zhou D, Du Q, Liang Q, Wang Q, Fang L, Wang G, Fan Q, Liu B, Zhou J, et al: Aberrant production of soluble inducible T-cell co-stimulator (sICOS) and soluble programmed cell death protein 1 (sPD-1) in patients with chronic hepatitis C. Mol Med Rep 7: 1197-1202, 2013

25. Song MY,Park SH, Nam HJ, Choi DH and Sung YC: Enhancement of vaccine-induced primary and memory $\mathrm{CD} 8(+) \mathrm{T}$-cell responses by soluble PD-1. J Immunother 34: 297-306, 2011.

26. Riganò R, Buttari $B$, De Falco E, Profumo E, Ortona E, Margutti P, Scottà C, Teggi A and Siracusano A: Echinococcus granulosus-specific T-cell lines derived from patients at various clinical stages of cystic echinococcosis. Parasite Immunol 26: 45-52, 2004.

27. Daneshvar H, Burchmore R, Hagan P and Phillips RS: Leishmania major $\mathrm{H}$-line attenuated under pressure of gentamicin, induces a Th1 response which protects susceptible $\mathrm{BALB} / \mathrm{c}$ mice against infection with virulent L. major. Parasitology 136: 1243-1250, 2009.

28. Rogan MT: T-cell activity associated with secondary infections and implanted cysts of Echinococcus granulosus in BALB/c mice. Parasite Immunol 20: 527-533, 1998.

29. Veldhoen M, Uyttenhove C, van Snick J, Helmby H, Westendorf A, Buer J, Martin B, Wilhelm C and Stockinger B: Transforming growth factor- $\beta$ 'reprograms' the differentiation of T helper 2 cells and promotes an interleukin 9-producing subset. Nat Immunol 9: 1341-1346, 2008.

30. Kimura A and Kishimoto T: IL-6: Regulator of Treg/Th17 balance. Eur J Immunol 40: 1830-1835, 2010

31. Hoffmann KF, Cheever AW and Wynn TA: IL-10 and the dangers of immune polarization: Excessive type 1 and type 2 cytokine responses induce distinct forms of lethal immunopathology in murine schistosomiasis. J Immunol 164: 6406-6416, 2000.

32. Lechner CJ, Grüner B, Huang X, Hoffmann WH, Kern P and Soboslay PT: Parasite-specific IL-17-type cytokine responses and soluble IL-17 receptor levels in Alveolar Echinococcosis patients. Clin Dev Immunol 2012: 735342, 2012. 